\title{
Left Atrial Myxoma with Mild Left Ventricular Dysfunction- A Case Report
}

\author{
Meher $\mathrm{T}^{1}$, PalashSMAZN ${ }^{2}$, Hasan $\mathrm{MK}^{3}$, Khan $\mathrm{TMNS}^{4}$, Zahangir $\mathrm{NM}^{5}$, HaiderMZ $^{6}$, Khan $\mathrm{SI}^{7}$, \\ Devnath $\mathrm{CK}^{8}$, Ahmed N${ }^{9}$, Ahmed $\mathrm{S}^{10}$
}

\begin{abstract}
Atrial Myxoma is the most common primary cardiac tumors accounting for about $50 \%$ of benign primary cardiac tumors, with the majority located in the left atrium. This is a case of large left atrial (LA) myxoma presented with features of mitral stenosis associated with moderate left ventricular failure (LVF) and mild pulmonary artery hypertension (PAH) The patient improved markedly after tumor excision.
\end{abstract}

\section{Keywords}

Myxoma, Left Atrium, Mitral Valve Obstruction, Ventricular dysfunction

\section{Introduction}

Myxomas are the most common type of cardiac tumors, accounting for $50 \%$ to $60 \%$ of the total in some case series, with an estimated incidence between 0.5 and 1 case per one million inhabitants per year. ${ }^{1}$ Over $70 \%$ of all cardiac myxoma originate from the left atrium (LA) and 18\% from the right atrium (RA). Biatrial myxoma account for $<2.5 \%$ of all cardiac myxoma. ${ }^{2}$ The symptoms have varied greatly, depending on the size and the localization of this tumor. Most present with one or more effects of a triad of constitutional, embolic and obstructive manifestations. The most common clinical presentation mimics that of mitral valve disease - either stenosis due to tumor prolapse into mitral orifice or regurgitation due to tumor induced valve trauma. Myxoma may present with heart failure with or without any obstructive coronary artery disease. We present a case of LA myxoma presented with features of mitral stenosis and moderate LV dysfunction without coronary artery disease.

\section{Case Report}

A 60-year-old diabetic, hypertensive woman with history of peripheral vascular disease with amputation of right $3 \mathrm{rd}$ toe and history of thyroidectomy for goiter 30 years back was admitted with exertional dyspnea and palpitations for the last 6 months.Constitutional symptoms such as arthralgia, myalgia, and weight loss were also present. Patient was non anemic, not icteric and had no clubbing, cyanosis and edema.

1. Registrar-Cardiothoracic \& Vascular Surgery, Apollo Hospitals Dhaka 2. Specialist- Cardiothoracic \& Vascular Surgery, Apollo Hospitals Dhaka 3. Registrar- Cardiothoracic \& Vascular Surgery, Apollo Hospitals Dhaka 4. Registrar-Cardiothoracic \& Vascular Surgery, Apollo Hospitals Dhaka 5. Associate consultant, Cardiothoracic \& Vascular Surgery, Apollo Hospitals Dhaka 6. Consultant \& Coordinator-Cardiothoracic \& Vascular Surgery, Apollo Hospitals Dhaka 7. Specialist-Cardiac Anaesthesia, Apollo Hospitals Dhaka 8. Sr. Registrar-Cardiac Anaesthesia, Apollo Hospitals Dhaka 9. Consultant \& Coordinator-Cardiothoracic Anaesthesia, Apollo Hospitals Dhaka 10. Consultant-Cardiothoracic \& Vascular Surgery, Apollo Hospitals Dhaka 
and edema. Pulse was irregular (78 bpm) with ectopic beats, and blood pressure was 130/80 $\mathrm{mmHg}$. On auscultation of precordium,loud first heart sound, low pitched "tumor plop" and a mid-diastolic murmur at apex were the characteristic findings masquerading as rheumatic mitral stenosis. ECG revealed premature ventricular contraction (PVC) and features of left bundle branch block (LBBB) (Fig.1). Chest $\mathrm{X}$-ray revealed mild cardiomegaly. Echocardiography revealed a large myxoma of $12 \mathrm{sq} . \mathrm{cm}$ in size occupying in the left atrium, which was

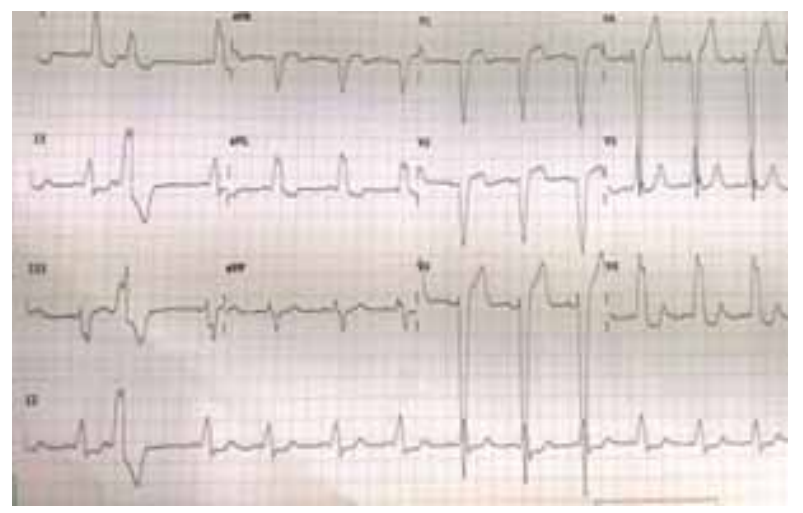

Fig. 1: ECG

which was attached to inter atrial septum by a stalk and move towards left ventricle during diastole, producing mitral valve obstruction. All the cardiac chambers were normal in dimension. Interventricular septum was hypokinetic and paradoxical in motion. Moderate LV systolic dysfunction was present. Ejection fraction (EF) was $40 \%$. There was Grade I mitral regurgitation (MR) - and mild pulmonary artery hypertension (PAH), Pulmonary artery systolic pressure (PASP) was $40 \mathrm{~mm} \mathrm{Hg}$.
Considering left ventricular dysfunction, coronary angiography was performed which revealed normal epicardial coronary arteries. The patient was scheduled for open heart surgery to excise left atrial myxoma. Anesthesia was induced without any incident. Midline sternotomy was done and cardiopulmonary bypass was instituted by doing aortic and bicaval cannulation. Right atrium opened by longitudinal incision. Interatrial septum was incised. Gelatinous myxoma connected by a stalk to the left side of interatrial septum was found. Myxoma was carefully

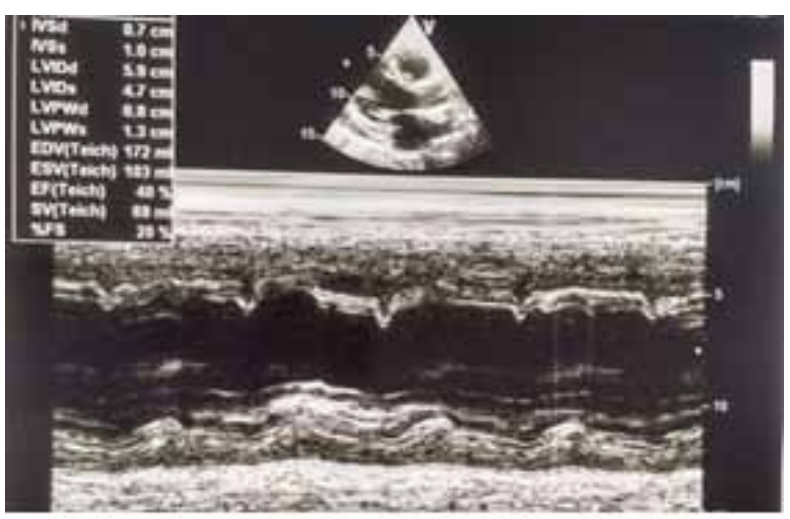

\section{Fig. 2: Echo showing the different cardiac chamber with myxoma in LA}

found. Myxoma was carefully scooped out. Stalk of the myxoma carefully resected out and cauterized with diathermy. LA cavity was thoroughly checked for any residual and washed thoroughly with normal saline. Then interatrial septum was closed by suturing with $5 / 0$ proline and RA was closed in single layer by continuous stich with $6 / 0$ proline. Patient was successfully weaned from cardiopulmonary bypass and de-cannulation done. Rest of the procedure was done in usual manner. Patient was shifted to intensive care unit (ICU) after completion of 
operation. Patient was recovered nicely with minimum inotropic support. Extubation was done after 12 hours. On 3rd post-operative day, patient was shifted from ICU to ward. Histopathological examination showed a tumor composed of round, polygonal or stellate cells arranged in clusters in a myxoid stroma admixed with blood clot and fibrin mass consistent with myxoma. Echocardiography on 5th postoperative day shows IVS is hypokinetic, paradoxical, MR grade I, mild AR, Aortic cusps

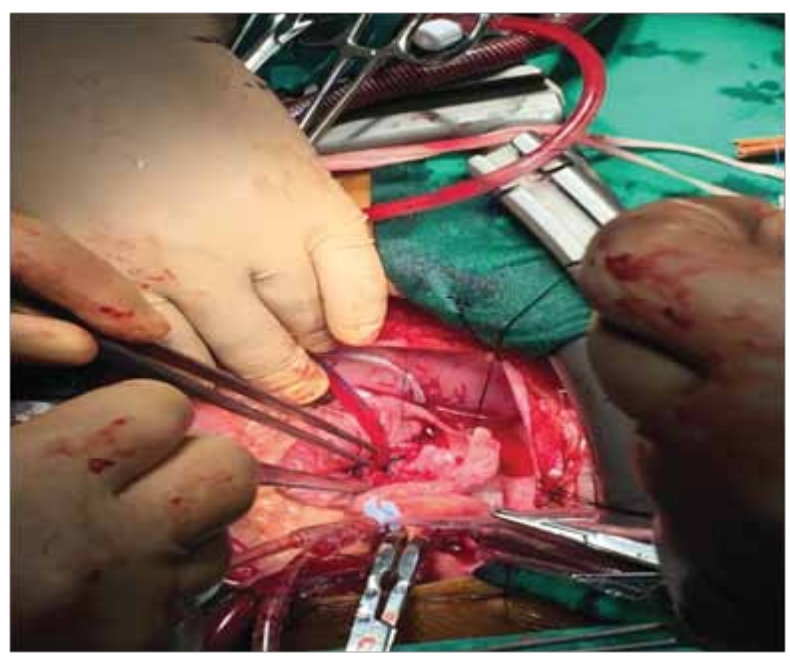

Fig.3:LA myxoma being scooped

are mildly thickened. All chambers are normal in dimension. Mild LV systolic dysfunction. All the valves are normal in morphology and motion.No intracardiac mass, vegetation or thrombus was seen. No pericardial effusion. LVEF- $45 \%$. On 7 th postoperative day she was discharged from hospital in a stable hemodynamic condition. On subsequent follow up in OPD patient was found clinically and hemodynamically stable and no signs of recurrence.

\section{Discussion}

The most common primary cardiac tumor is the benign myxoma, which in the large majority of cases is solitary. The mean age of presentation with sporadic myxoma is 56 years and $70 \%$ are female. ${ }^{3}$ Risk factors for atherosclerosis such as systemic hypertension, dyslipidemia and coronary artery disease is also higher in this age range. Prevalence of coronary artery disease in patients with myxoma has been reported between $20.3 \%$ and $36.6 \%$.

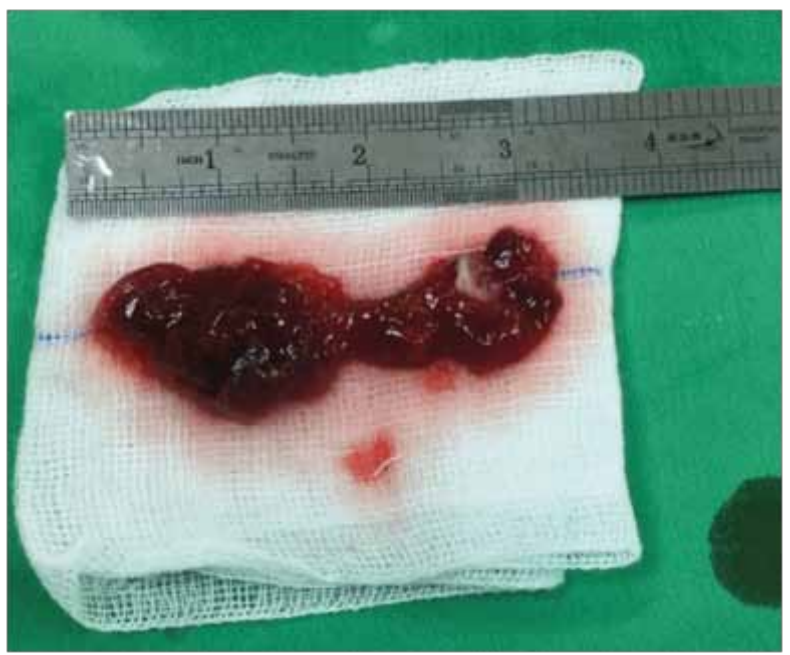

Fig.4: LA myxoma after excision out

Myxoma has variable presentation. In a study of cardiac myxoma on 49 patients it was shown that myxoma produced a prolapse into the left ventricle in $40.8 \%$ of the patients, presented with features of mitral stenosis in $10.2 \%$ and threatened left ventricular outflow tract obstruction in $2.0 \% .{ }^{5}$ Sudden deaths may also occur.

Few case study shows that myxoma may present with heart failure. Coronary embolization of tumor fragments causing myocardial ischemia and coexisting coronary atherosclerosis are the 
and coexisting coronary atherosclerosis are the recognized reasons of $\mathrm{LV}$ dysfunction in the patient with left atrial myxoma. ${ }^{6}$ Boutayeb et al (2013). described RA myxoma in patient with severe LV dysfunction. Author hypothesized that coronary sinus occlusion by myxoma prolapse during the diastole was causing impaired left ventricular function. ${ }^{7}$

In our case we have found LA myxoma was associated with LV dysfunction and septal wall hypokinesia and paradoxical movement without any obstructive coronary artery disease. Chockalingam et al (2006). suggested cardio-depressant effect of LA myxoma through unclear mechanisms that reversed only after myxoma removal ${ }^{8}$. Very few studies suggest that overproduction of IL-6 by cardiac myxoma could be a possible cause of its association with LV dysfunction. ${ }^{9}$ Yan et al (2010). confirmed the finding that IL-6 is not only a biomarker predicting the onset of heart failure but also potentially linked in the pathophysiological cascade, possibly through nitroso-redox imbalance and other direct mechanisms especially affecting the septal and inferior wall of left ventricle. ${ }^{10}$

Raut, Shad and Maheshwari et al (2016) reported a case of a left atrial myxoma with severe ventricular dysfunction without any obstructive coronary artery disease. They hypothesized undiagnosed concomitant dilated cardio- myopathy or unknown cardio-depressant effect of myxoma may be the cause of heart failure and suggested further research. ${ }^{11}$

Dixit et al (2017) reported 2 cases of myxomas. In the 1st case they noticed biatrial myxoma associated with severe biventricular failure with severe septal, inferior, and inferolateral hypokinesia and biventricular dysfunction without any coronary artery disease. In the 2nd case they noticed LA myxoma associated with global hypokinesia and severe biventricular failure with mild TR and MR. In both the patients, regional wall motion abnormalities reduced significantly after excision of myxomas and such early response points toward the removal of a causative element. ${ }^{12}$

In our case, LV dysfunction was moderate associated with mild MR and mild PAH. After sugical excision of the tumour though LV function improved slightly but marked clinical and hemodynamic improvement was observed.

\section{Conclusion}

Surgical removal of the tumor should be performed as soon as possible. Long-term prognosis is excellent and recurrences are rare. In follow-up examinations as well, echo- cardiography is essential to evaluate improvement of LV function. Further insight into the definite cause of ventricular dysfunction in patient with LA myxoma is needed.

\section{References}

1. McManus B. Primary tumors of the heart. In: Mann DL, Douglas PZ, Libby P, Bonow RO, editors. Braunwald' s Heart Disease, a Text Book of Cardiovascular Medicine. 9th ed. Philadelphia: Elsevier Saunders; 2012.p. 1638-50.

2. Irani AD, Estrera AL, Buja LM andSafi HJ. Biatrial myxoma: A case reportand review of the literature. Journal of Cardiac Surgery, 2008; 23:385-90.

3. Erdil N, Ates S, Cetin L, Demirkilic U, Sener E and Tatar H. Frequency of leftatrial myxoma with concomitant coronary artery disease. Surgery Today,2003; 33(5): 328-31.

4. Li AH, Liau CS, Wu CC, Chien KL, Ho YL, Huang CH, et al. Role of coronary angiography in myxoma patients: a 14-year experience in one medical center. Cardiology, 1999; 92:232-35. 
5. Keelinga IM,Oberwaldera $\mathrm{P}$, Anelli-Montia M, Schuchlenzb H, Demelc U, Tilzc GP, et al. Cardiac myxomas: 24 years of experience in 49 patients, European Journal of Cardio-thoracic Surgery, 2002; 22(6): 971-7.

6.Gismondi RAOC, Kaufman R, Correa GADCP, Nascimento C, WeitzelLH, Reis JOB, et al.Left Atrial Myxoma Associated with Obstructive Coronary Artery Disease, Arq Bras Cardiol, 2007; 88(1): e1-e2

7. Boutayeb A, Marmade L, Bensouda A andMoughil S. Right atrial myxoma and severe left ventricular dysfunction: which explanations? Which management? Heart Lung Circulation, 2013; 22:309-11.

8. Chockalingam A, Jaganathan V, Gnanavelu G, Dorairajan S andChockalingam V. Severe left ventricular dysfunction in left atrial myxoma - report of 2 cases. Angiology. 2006;57(1): 119-22.
9. Mendoza CE, Rosado MF and Bernal L. The role of interleukin-6 in cases of cardiac myxoma. Clinical features, immunologic abnormalities, and apossible role in recurrence. Texas Heart Institute Journal, 2001;28(1):3-7.

10. Yan AT, Yan RT, Cushman M, Redheuil A, Tracy RP, Arnett DK, et al.Relationship of interleukin-6 with regional and global left-ventricularfunction in asymptomatic individuals without clinical cardiovasculardisease: Insights from the Multi-Ethnic Study of Atherosclerosis. European Heart Journal, 2010;31:875-82.

11. Raut MS, Shad S an dMaheshwariA. Left atrial myxoma with biventricular dysfunction. Indian heart journal, 2016;68: s163- 5 .

12. Dixit A, Tewari P, Soori R and Agarwal SK. Atrial myxomas causing severe leftand right ventricular dysfunction. Annals of Cardiac Anaesthesia, 2017;20:450-2. 